\title{
Las tesis doctorales en España (1997-2008): análisis, estadísticas y repositorios cooperativos
}

\author{
Eulàlia Fuentes Pujol*, Llorenç Arguimbau Vivó ${ }^{\star *}$
}

\begin{abstract}
Resumen: El artículo analiza el estado de la producción y difusión de tesis doctorales en las universidades españolas, a la luz de los cambios acaecidos en el período 1997-2008. Entre los factores de transformación cabe señalar el proceso de adaptación al Espacio Europeo de Educación Superior (EEES), el enorme impacto de las Tecnologías de la Información y de las Comunicaciones (TIC) y el movimiento orientado al acceso abierto (Open Access) de los documentos científicos. Después de una exhaustiva revisión bibliográfica de la literatura profesional sobre tesis doctorales, se examinan las series estadísticas elaboradas por el Instituto Nacional de Estadística (INE), EUROSTAT y otras fuentes especializadas. A continuación, se describen algunas de las principales bases de datos y repositorios cooperativos de tesis existentes a nivel español e internacional. Finalmente, se analizan los datos obtenidos y se extraen algunas conclusiones sobre la capacidad del sistema académico español para generar y difundir conocimiento a partir de la formación de nuevos investigadores.
\end{abstract}

Palabras clave: Tesis doctorales, repositorios, cooperación, universidad, España.

\section{Doctoral theses in Spain (1997-2008): analyses, statistics and cooperative repositories}

Summary: This paper discusses the state of production of doctoral theses in Spanish universities and their dissemination, in light of changes that occurred over the period 1997-2008. The transformation factors that had to be dealt with included the process of adaptation to the European Higher Education Area (EHEA), the buge impact of Information and Communication Technologies (ICT), and the appearance of the Open Access movement designed to provide free access to scientific and scholarly literature. Following an exhaustive review of the existing studies on doctoral theses, this paper examines the statistics compiled by the National Statistics Institute (INE), EUROSTAT and other specialized sources. Some of the major databases and cooperative theses repositories at both the Spanish national and international level are described. Finally, the data are analysed and conclusions drawn on the ability of the Spanish academic system to disseminate knowledge via the training of new scientists.

Keywords: Doctoral theses, repositories, cooperation, university, Spain.

* Àrea de Documentació. Universitat Autònoma de Barcelona. Correo-e: eulalia.fuentes@uab.cat

** Observatori de la Recerca. Institut d'Estudis Catalans. Barcelona. Correo-e: llarguimbau@iec.cat Área de Documentació. Universitat Autónoma de Barcelona. Correo-e llorenc.arguimbau@uab.cat

Recibido: 23-12-09; 2. ${ }^{a}$ versión: 26-8-09; 3. ${ }^{a}$ version: 24-9-09; aceptado: 9-10-09. 


\section{Introducción}

Una tesis doctoral supone la culminación del ciclo de aprendizaje e investigación de un científico en formación bajo la dirección de uno o varios doctores. Una vez superada la defensa pública de la investigación ante un tribunal de expertos en la materia, el doctorando obtiene el título de doctor en su especialidad y, a partir de ese momento, tiene la posibilidad de difundir el conocimiento generado a través de la publicación de la tesis o de otros documentos (artículos, comunicaciones, etc.). Antes de la expansión de Internet, las tesis doctorales formaban parte de la literatura gris ya que su difusión y acceso estaban muy limitados (Merlo y Sorli, 2002; Miguel, 2000; Moralejo, 2000; Orera, 2003a). Actualmente para el avance de la ciencia tiene cada vez mayor importancia promover la comunicación científica a todos los niveles (Agudelo y otros, 2003).

En tanto que son trabajos originales y muy especializados que aportan nuevos conocimientos en su área, cabe reivindicar la importancia científica de las tesis ya que proporcionan una imagen fiel de las nuevas vías de investigación (Muñoz, 2005), además de la exhaustiva revisión bibliográfica que suele acompañar este tipo de trabajos (Miguel, 2000). Como indicadores, resultan útiles para calibrar el potencial de formación de nuevos investigadores de un sistema, así como la productividad científica de los directores (Buela-Casal, 2005; Moyano y otros, 2006).

El presente artículo analiza el estado actual de la producción y difusión de tesis doctorales en las universidades españolas, a la luz de los cambios acaecidos. En el período 1997-2008. Es conveniente describir el escenario actual para calibrar la situación del sistema español, identificar patrones singulares y debatir tendencias de futuro. A continuación se comentan los principales factores de transformación, entre los cuales hay que tener en cuenta el proceso de adaptación al Espacio Europeo de Educación Superior (EEES), el impacto de las Tecnologías de la Información y de las Comunicaciones (TIC) y, por último, el movimiento orientado al acceso abierto (Open Access) de los documentos científicos.

La adaptación al EEES se asienta sobre los fundamentos jurídicos establecidos por el RD 1393/2007, promulgado con el objetivo de adaptar los estudios españoles de postgrado a las nuevas directrices europeas. Entre otras innovaciones, cabe destacar la posibilidad de obtener la mención de doctor europeo o la presentación de tesis doctorales como compendio de publicaciones. En el modelo anterior (reglamentado por el RD 778/1998) los estudios de doctorado incorporaban una parte de docencia y otra de investigación, mientras que el nuevo modelo parece orientarse más decididamente hacia las tareas de formación y desarrollo científico, que culminan en la tesis doctoral, definida como un trabajo original de investigación.

Por otro lado, la edición digital de las tesis y su posterior tratamiento en catálogos, bases de datos y repositorios comienza a solucionar los problemas de acceso a estos documentos (Orera, 2003a). En este sentido, la publicación parece el mejor sistema para garantizar la accesibilidad. Al margen de la vía comercial, 
los soportes de publicación han ido evolucionando (fotocopia, microficha, CDROM, etc.), hasta alcanzar el salto cualitativo que supone la combinación sinérgica de la producción original en formato electrónico, la tecnología web y las bibliotecas digitales. A pesar de algunos problemas pendientes (estándares, propiedad intelectual, soportes físicos, etc.), el crecimiento de los repositorios digitales queda justificado por sus múltiples ventajas (Orera, 2003a): reducción de costos, sobretodo en proyectos cooperativos; publicación inmediata; aumento de la visibilidad internacional; mejor calidad de los servicios bibliotecarios, etc.

Por su parte, el movimiento de acceso abierto a la ciencia ha emergido en España con múltiples proyectos, sobre todo, a partir de finales de 2004 (Melero y otros, 2008). Así diversas universidades y organismos científicos españoles se están dotando de depósitos digitales abiertos (institucionales y cooperativos), donde las tesis doctorales también están presentes.

\section{Objetivos, metodología y revisión bibliográfica}

Los dos objetivos básicos de este artículo son: $a$ ) recopilar y analizar los datos estadísticos fundamentales sobre tesis doctorales aprobadas en España desde el año 1997; b) describir algunas de las principales bases de datos y recursos cooperativos nacionales e internacionales usados para difundir específicamente las investigaciones doctorales españolas. De esta forma, la comparación de los datos estadísticos con las fuentes especializadas permite determinar la capacidad del sistema académico español para formar nuevos investigadores, así como el grado de accesibilidad a las tesis doctorales.

A nivel metodológico, el presente estudio se fundamenta en los datos cuantitativos obtenidos a partir de los institutos estadísticos oficiales y de los recursos digitales de tesis doctorales (Apartado 3), presentados de forma conjunta y sintética. Para facilitar el análisis de los datos, el período estudiado se inicia en el curso 1997-1998 y finaliza en el 2007-2008. A su vez, la mayoría de las consultas se han realizado durante el mes de junio de 2009, para que los datos sean lo más actualizados posibles.

Como paso previo al estudio, se ha efectuado una extensa revisión bibliográfica de la literatura profesional sobre tesis doctorales en España publicada desde el año 1997. La bibliografía de este artículo es el resultado de la búsqueda exhaustiva en las siguientes fuentes de información: Bases de datos bibliográficas del CSIC; E-prints in Library and Information Science (E-LIS); ISI Web of Knowledge; Library, Information Science \& Technology Abstracts (LISTA); TESEO: Base de datos de tesis doctorales; y, por último, Trobador (metabuscador de recursos electrónicos de las bibliotecas de la Universidad Autónoma de Barcelona (UAB)).

El estudio detallado de la literatura profesional sobre tesis en España excede los límites de este artículo, pero a continuación se destacan brevemente los aspectos más relevantes que se han podido observar. Además de algunas tesis doctorales (Gete, 2005; Martínez, 2007; Pérez, 2007; Rorralbo, 2001; Sabater, 2001; 
Urbano, 2000a), básicamente se han recuperado artículos de revista que adoptan una perspectiva general (Merlo y Sorli, 2002; Miguel, 2000; Moralejo, 2000; Orera, 2003a, 2003b; Villarroya, 2004), de género (Muñoz, 2005; Villarroya, 2008), o especializada en un ámbito determinado.

En concreto, existen artículos sobre las siguientes áreas temáticas:

Archivística (Bonal, 2000); Bibliometría (Alcaín y Ruiz-Galvez, 1997; Delgado y otros, 2006); Comunicación (Jones y Baró, 1997); Documentación (Fuentes y González, 2002; Lopez, 2002a; 2002b; Lopez y otros, 2005a; 2005b; Orera, 2003b; 2004; Zapico, 2002); Econometría (Guisan y Expósito, 2007); Educación (Fernández y otros, 2003; 2008; Ponce y otros, 1998; Vallejo y otros, 2006); Feminismo (Torres y Torres, 2005); Geografía (Hernández, 1999; Lázaro, 2002); Informática (Urbano, 2000b); Lingüística (Pablo, 2006); Medicina (Centeno y otros, 2001; Diego y Prim, 2008; Figueredo y otros, 2002; Muñoz y Peña, 1998; 2000); Musicología (Nagore, 2005); Ornitología (Barbosa, 2000); Psicología (Agudelo y otros, 2003; Civera y Tortosa, 2001; Fernández, 2004); Publicidad (Martínez, 2004); Química (Vallmitjana y Sabaté, 2008); Relaciones Públicas (Castillo y Xifra, 2006); Turismo (Ortega y Rodríguez, 2004).

Los estudios sectoriales sobre la producción doctoral de áreas concretas pretenden determinar sus rasgos característicos, evolución y grado de consolidación. La fuente de información más habitual es la base de datos TESEO, complementada en ocasiones por otras bases de datos, registros administrativos, catálogos de biblioteca, entrevistas a expertos, revistas especializadas, etc. A partir de los datos recopilados, las dimensiones de análisis más comunes son los indicadores temáticos (disciplinas y áreas de estudio) y de productividad (directores, tribunales, años, universidades y centros de lectura). En algunos casos se introducen criterios de ponderación (Agudelo y otros, 2003), como la antigüedad de la universidad, el número de docentes, etc. A nivel metodológico, el estudio cuantitativo se puede complementar, en primer lugar, con los análisis de redes sociales asociadas a la detección de escuelas científicas (Delgado y otros, 2006; López, 2002a; 2002b) y, en segundo lugar, con los análisis de citaciones derivados de la revisión bibliográfica exhaustiva que conlleva una tesis doctoral (Fernández y otros, 2003; Urbano, 2000b; Vallejo y otros, 2006; Vallmitjana y Sabaté, 2008).

(Dada la cantidad de siglas y acrónimos utilizados, se ha considerado pertinente añadir un anexo, con esta información, al final del artículo).

\section{Fuentes de información estadística}

A continuación, se describen las series estadísticas oficiales sobre alumnos de doctorado y tesis, elaboradas por el Instituto Nacional de Estadística (INE) (http://www.ine.es) y por EUROSTAT (http://epp.eurostat.ec.europa.eu), a nivel europeo. Respecto a estas dos grandes fuentes de información, los dos institutos facilitan la metodología utilizada, el diseño de informes a medida del usuario y los datos obtenidos son exportables para su posterior tratamiento. Ahora bien, la 
comparación de las estadísticas se ve dificultada por las diferencias en la cobertura (1988-2008 en el INE y 2004-2007 en EUROSTAT), los períodos de tiempo (cursos académicos en el INE y años naturales en EUROSTAT), y la clasificación temática ( 5 y 9 áreas respectivamente).

También se mencionan otras fuentes de información especializada nacionales e internacionales, como el informe bianual de la Conferencia de Rectores de las Universidades Españolas (CRUE) (http://www.crue.org), la Secretaría General del Consejo de Coordinación Universitaria (CUC) (http://univ.micinn.fecyt.es/univ/ ccuniv), el portal Statistics (http://www.oecd.org/statsportal) de la Organización para la Cooperación y el Desarrollo Económico (OCDE) y el Institute for Statistics de la UNESCO (http://www.uis.unesco.org).

Finalmente se analizan los resultados obtenidos.

\subsection{Instituto Nacional de Estadística (INE)}

La finalidad de la Estadistica de enseñanza universitaria consiste en conocer las características más relevantes del alumnado, personal docente y centros (propios o adscritos), para disponer de una panorámica global de la educación superior en España. Además, esta información sirve para cumplimentar los cuestionarios requeridos por los diferentes organismos internacionales (EUROSTAT, OCDE, UNESCO, etc.). A comienzos de marzo del curso académico, se envía la solicitud oficial a cada centro universitario para recoger la información de base. A través de la consulta en línea del INE, se puede acceder a la serie estadística sobre el tercer ciclo universitario desde el curso 1988-1989 hasta el 2007-2008, que incluye datos sobre el alumnado matriculado en doctorado y sobre las tesis doctorales aprobadas. El período estudiado se inicia en el curso 1997.

\subsection{EUROSTAT}

En base a los datos suministrados por los diferentes institutos nacionales, EUROSTAT elabora estadísticas para la Unión Europea (UE27). En el caso de los estudios de doctorado, los datos sobre alumnos y titulados son accesibles, respectivamente, para el período 2006-2007 y 2004-2007. Las estadísticas se pueden consultar desde diferentes perspectivas: campo educativo; área geográfica (agregados europeos y estados miembros); nivel educativo (según la International Standard Classification of Education 1997, ISCED97), etc.

\subsection{Otras fuentes estadísticas}

En el marco de la mejora del sistema de información universitaria, la CRUE (http://www.crue.org) ha impulsado cinco ediciones del informe bianual La universidad española en cifras. Publicada en septiembre de 2008, la última edición 
contiene información académica, productiva y financiera del curso 2006-2007 (datos presupuestarios de 2006). Como novedades, esta edición incorpora información sobre las universidades privadas y un bloque cualitativo de indicadores universitarios. Por otro lado, la Secretaría General del CUC (http://univ.micinn. fecyt.es/univ/ccuniv) también ofrece un apartado de Estadística universitaria con datos generales, estadísticas de alumnos y personal e información sobre indicadores del sistema universitario, incluidos los correspondientes al tercer ciclo.

A su vez, el portal Statistics (http://www.oecd.org/statsportal) de la OCDE y el Institute for Statistics de la UNESCO (http://www.uis.unesco.org), permiten la comparación mundial de los datos básicos sobre el ciclo universitario superior.

\subsection{Análisis de los datos de las fuentes de información estadística}

El análisis de los datos estadísticos del INE (Tablas I y II) pone de manifiesto que la evolución global de alumnos de doctorado y de tesis doctorales aprobadas es muy parecida: asciende hasta alcanzar las mejores cifras en el curso 2003-2004, para descender posteriormente. Entre otras hipótesis, una causa del actual descenso podría ser debido a las modificaciones de la normativa de doctorado.

La distribución por áreas de las tesis españolas, tanto por el alumnado matriculado (Tabla I), como por las tesis doctorales aprobadas (Tabla II), también muestra datos interesantes. Globalmente, en el período 1997-2008 el área de Ciencias Experimentales y de la Salud (32,6\% de alumnos matriculados y 47,0\% de tesis aprobadas), ocupa la primera posición, seguida por el área de las Ciencias Sociales y Jurídicas $(28,2 \%$ de alumnos matriculados y $22,5 \%$ de tesis aprobadas), juntas representan un $60 \%$ de alumnos matriculados y un $47 \%$ de tesis aprobadas. Les sigue el área de las Humanidades (20,5\% de alumnos matriculados y $14,4 \%$ de tesis aprobadas), que representa conjuntamente con las anteriores un $80 \%$ del alumnado matriculado y un $70 \%$ de tesis aprobadas. Finalmente, el área de Ingeniería y Tecnología (11,5\% de alumnos matriculados y 12,4\% de tesis aprobadas), a pesar de ser el área con mayor crecimiento, pasando de 687 tesis en el curso 1997-1998 a 1019 en el 2007-2008, queda la última en el ranking. Ésto puede ser debido a que en estos estudios, mayoritariamente, se realiza el proyecto fin de carrera, (que no deja de ser un trabajo importante de investigación) y que probablemente la incorporación al mercado de laboral es más rápida o que el mercado requiere más masters o postgrados de especialización y menos la obtención del título de doctor que en otros ámbitos.

En el período 1997-2008, el porcentaje de tesis doctorales leídas respecto al número de alumnos matriculados alcanza una media total de 9,9\%.

A su vez, el porcentaje de tesis doctorales aprobadas respecto al número total de alumnos matriculados registra un 14,3\% en las Ciencias Experimentales y de la Salud (248.424 matriculados y 35.664 tesis aprobadas), un 10,7\% en Ingeniería y Tecnología (87.551 matriculados y 9.369 tesis aprobadas), mientras que en Ciencias Sociales y Jurídicas un 7,9\% (215.042 matriculados y 17.048 tesis 

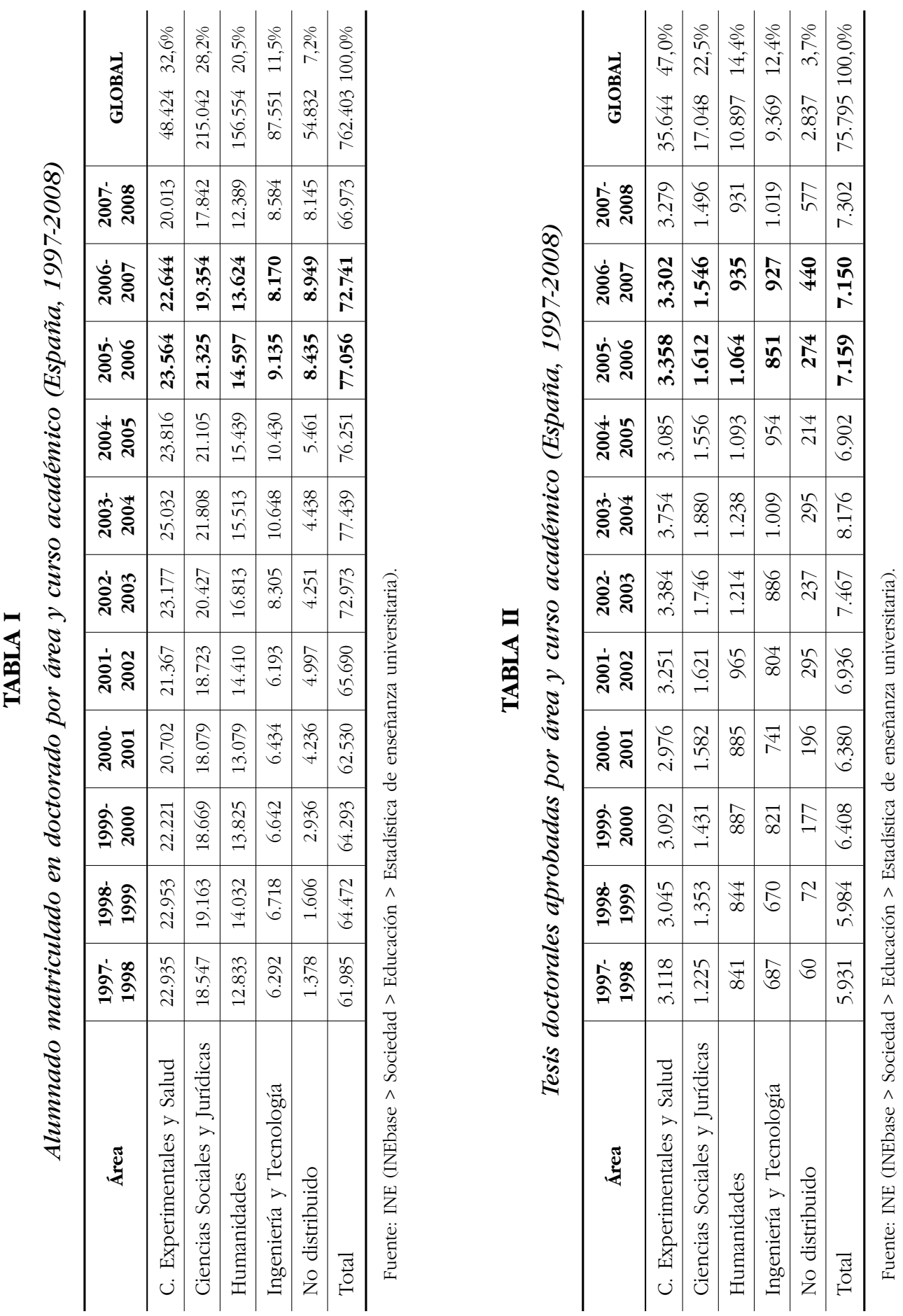
aprobadas) y en Humanidades un 6,9\% (156.554 matriculados y 10.897 tesis aprobadas).

Pero, en general, comparando las Tablas I y II, aunque se matriculen un número elevado de alumnos a los doctorados en las distintas áreas (762.403), las tesis leídas y aprobadas (75.795), se reducen aproximadamente a un 10\% de los alumnos matriculados. Hay muchos alumnos que pueden llegar a conseguir el grado de suficiencia investigadora, pero que posteriormente no realizan o no terminan la tesis doctoral empezada, por diversos motivos, inserción en el mundo laboral, no querer dedicarse a la investigación o a la docencia, etc.

A nivel europeo, sólo disponemos de los datos completos del bienio 20062007, de los alumnos matriculados (Tabla III) y de 2004 a 2007 para los nuevos doctores (Tabla IV), por campo educativo y área geográfica. Por ello solo analizaremos el bienio 2006-2007, donde el porcentaje de titulados respecto a los alumnos en la UE27 llega al 18,6\%. A partir de estos datos, se deduce que en un año o curso académico, en España, uno de cada 10 alumnos obtiene el título de doctor, mientras que en Europa esta proporción es prácticamente el doble.

También a nivel europeo la distribución global de tesis por áreas (Tabla IV) evidencia algunas peculiaridades del sistema español, ya que éste registra un porcentaje inferior en los sectores Engineering, Manufacturing \& Construction (8,2\% en España y 13,7\% en la UE27), Agriculture \& Veterinary (3,7\% y 4,1\%), y por último, Health E Welfare $(17,0 \%$ y $20,8 \%)$. Si bien un cuatrienio es un período de tiempo excesivamente breve, todas las áreas de la UE27 registran tasas de crecimiento positivo (un 16,5\% en global), mientras que en España las cifras son negativas (concretamente, el descenso en conjunto es del 12,5\%). La convergencia con Europa parece difícil a corto y medio plazo.

No obstante, si observamos las Tablas III y IV (2006 y 2007) y las comparamos con el mismo período de las Tablas I y II (2005-2006 y 2006-2007) por lo que respecta a España (ver columnas en negrita), aparentemente parece que los datos son muy distintos, pero analizando las Tablas III y IV (período 2006-2007) y agregando los campos educativos, presentados por EUROSTAT para que coincidan de la forma más aproximada posible con los del INE, vemos que los datos no son tan distintos y que los totales coinciden totalmente (Tablas V y VI).

\section{Bases de datos y repositorios cooperativos}

Debido a la importancia estratégica de las tesis como documentos científicos y culminación de los estudios de postgrado, existe la necesidad de promover normas generales e institucionales para su gestión, control, conservación, difusión y acceso. Moralejo (2000) considera que "los originales de las tesis entregadas en la universidad, una vez finalizados los trámites administrativos, deben reunirse para ser conservados en un depósito bibliográfico, único, que garantice su proceso técnico, su conservación, su difusión y el acceso a la información que contienen". En esta misma línea, el artículo 21.8 del RD 1.392/2007 estipula que "una 


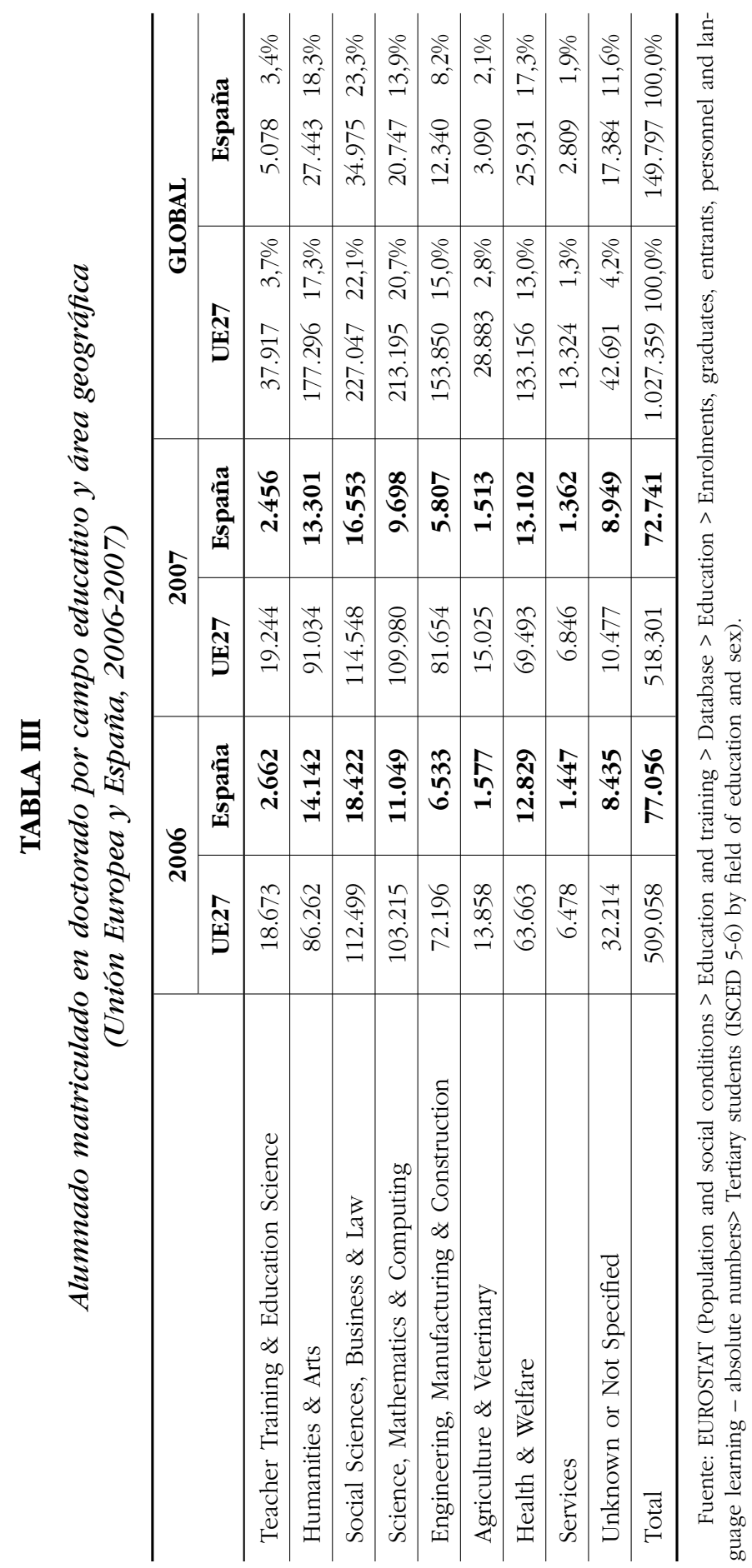

Rev. Esp. Doc. Cient., 33, 1, enero-marzo, 63-89, 2010. ISSN: 0210-0614. doi:10.3989/redc.2010.1.711 


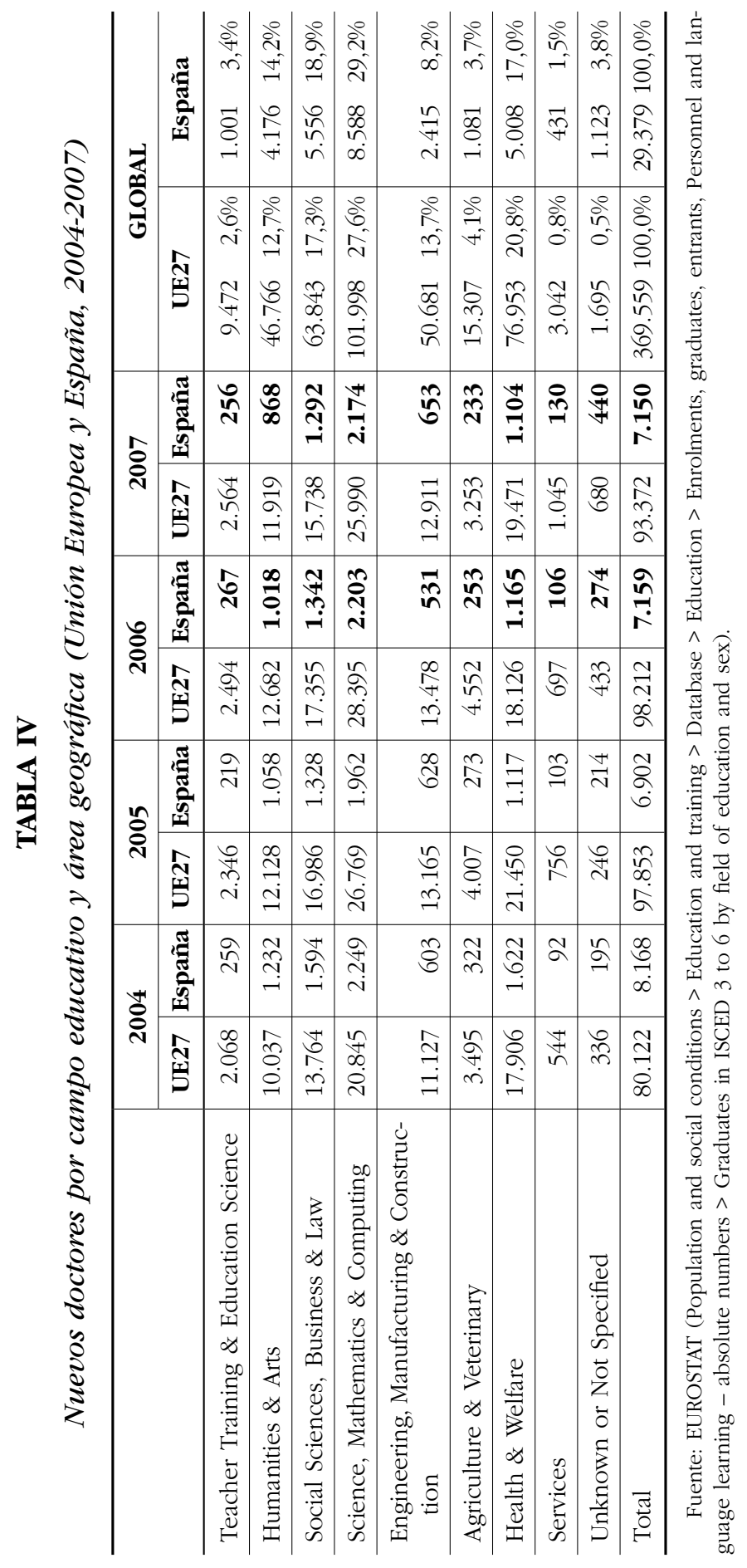




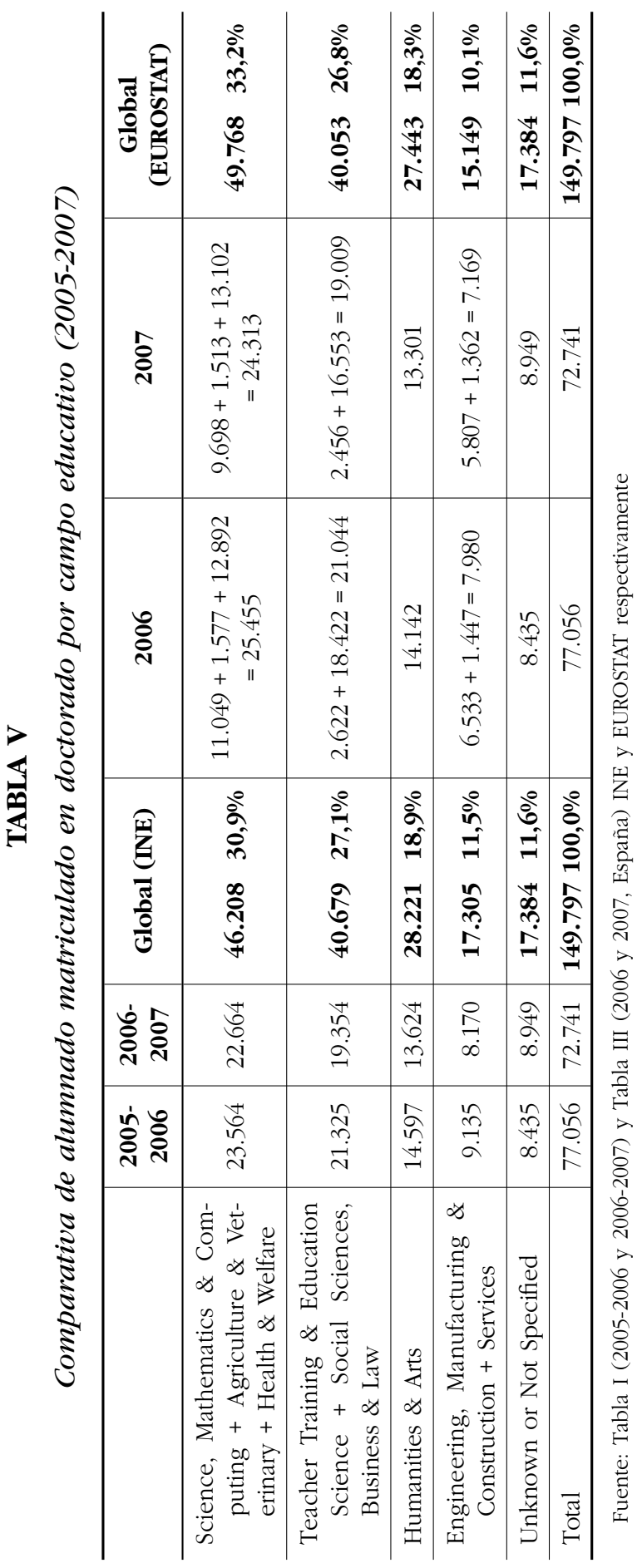




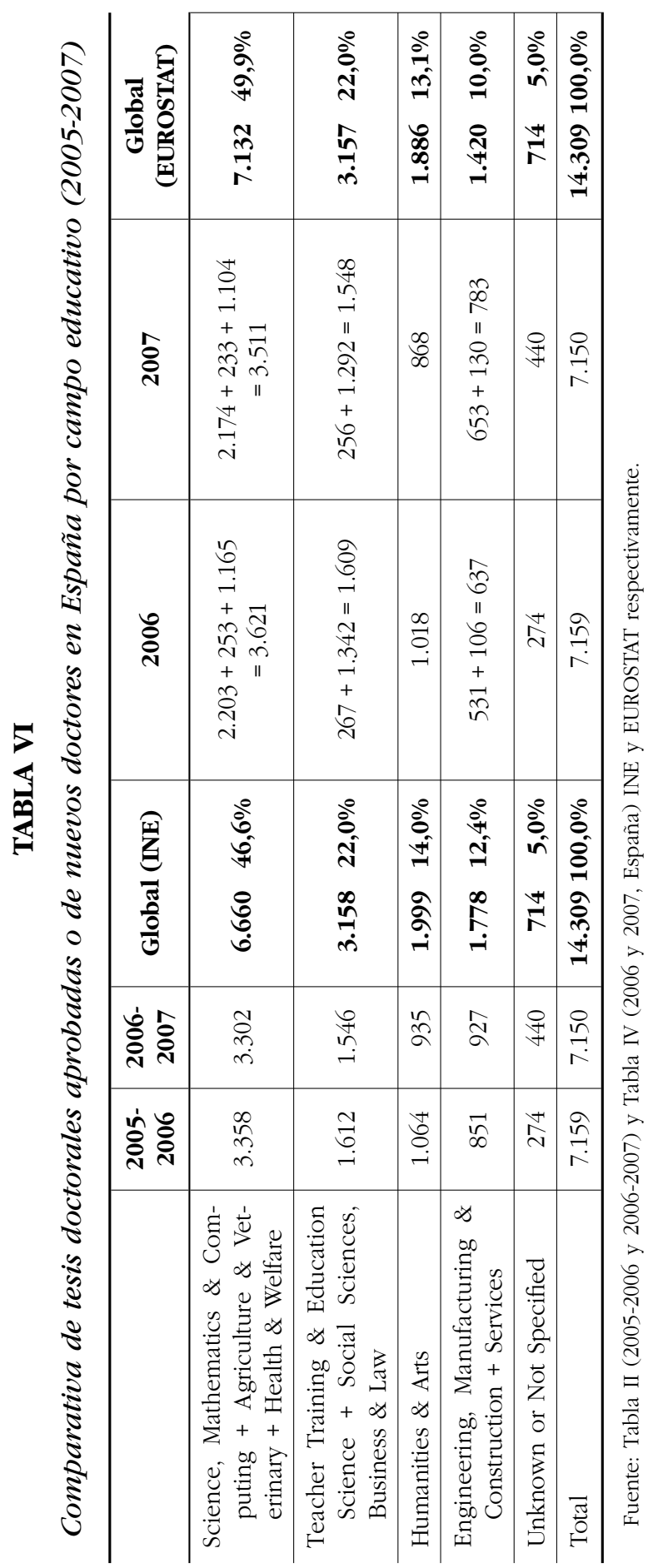


vez aprobada la tesis doctoral, la Universidad se ocupará de su archivo y remitirá un ejemplar de la misma así como la información necesaria al Ministerio de Educación y Ciencia a los efectos oportunos".

La digitalización y las redes de comunicaciones ofrecen posibilidades inmensas para la difusión del conocimiento científico. Cada vez más, las universidades españolas están impulsando el desarrollo de sistemas para difundir y acceder libremente a las tesis y otros documentos académicos mediante repositorios institucionales y cooperativos. En el marco del acceso abierto a la literatura científica, el protocolo Open Archives Initiative Protocol for Metadata Harvesting (OAI$\mathrm{PMH}$ ) facilita la construcción de sistemas que se alimentan de forma automática y descentralizada. Este protocolo de intercambio de información permite efectuar búsquedas centralizadas y conjuntas sobre los metadatos de los repositorios asociados, potenciando así la recuperación y el acceso a través de buscadores académicos internacionales, como por ejemplo, OAISter (http://www.oaister.org) .

El movimiento de acceso abierto a la ciencia ha emergido en España con múltiples proyectos, sobretodo a partir de finales de 2004 (Melero y otros, 2008). Según datos de OpenDoar (http://www.opendoar.org), a mediados de 2009 existen 44 repositorios españoles de acceso abierto, el 81,8\% de los cuales son institucionales, principalmente de universidades. Entre la amplia variedad de documentos recopilados (artículos de revista, comunicaciones, informes de investigación, working papers, etc.), las tesis están presentes en 22 de los 44 repositorios. A su vez, se pueden localizar 49 depósitos a través de Buscarepositorios (http://www.accesoabierto.net/repositorios), recurso promovido por el Grupo de investigación "Acceso abierto a la ciencia", formado por miembros del Consejo Superior de Investigaciones Científicas (CSIC), Universidad de Barcelona (UB) y Universidad de Valencia (UV).

Las universidades que trabajan en esta línea incorporan en los procedimientos asociados a la obtención del título de doctor el depósito de las tesis completas en repositorios digitales, previa autorización de los autores. Mediante un contrato de edición, el autor de la tesis autoriza su publicación en Internet, reservándose el derecho de publicarla en otras editoriales y soportes. Si se trata de una tesis por compendio de publicaciones, sólo se pueden difundir íntegramente los apartados acordados con las editoriales, además de los objetivos, metodología, resultados, etc.

Existen diferentes depósitos digitales y bases de datos referenciales sobre tesis a nivel español e internacional. A continuación se describen algunos de los principales recursos cooperativos específicamente dedicados a la difusión y acceso a tesis doctorales, dejando al margen los depósitos institucionales, comerciales o especializados en un ámbito concreto. También se excluye el importante papel de los catálogos de biblioteca en el control bibliográfico de este tipo de documentos, que podría dar lugar a otro artículo.

Por su importancia en el sistema español, también se trata la base de datos TESEO. 


\subsection{TESEO: Base de datos de tesis doctorales (https://www.micinn.es/ teseo). Ministerio de Ciencia e Innovación (MICINN)}

A mediados de los años setenta el Ministerio de Educación y Ciencia creó un fichero mecanizado de tesis doctorales. Actualmente, TESEO es la principal fuente de información sobre las tesis aprobadas en las universidades españolas desde 1976. La base de datos permite la búsqueda por múltiples criterios: autor, director, título, resumen, universidad, departamento, curso académico y palabras clave (en base a un tesauro). La última versión disponible está ideada como un sistema de gestión de tesis doctorales a nivel nacional. En este sentido, TESEO permite registrarse como doctorando y realizar el procedimiento completo desde que una tesis se da de alta hasta que, después de su lectura y aprobación, es publicada por los servicios universitarios competentes (generalmente, bibliotecas o servicios de publicaciones).

En el período analizado (1997-2008), TESEO ha registrado 65.854 tesis (Tabla VII). Comparando los datos de TESEO con los del INE del mismo período hay una diferencia significativa de 9941 tesis. Ello puede ser debido a distintas causas, como veremos en el análisis de los datos recogidos (Apartado 5).

\section{TABLA VII}

Comparativa de las tesis registradas por INE y TESEO (España, 1998-2007)

\begin{tabular}{c|c|c|c|c}
\hline Curso & Tesis INE & Tesis TESEO & Diferencia & Porcentaje \\
\hline $1997-1998$ & 5.931 & 5.741 & 190 & $96,8 \%$ \\
\hline $1998-1999$ & 5.984 & 5.195 & 789 & $86,8 \%$ \\
\hline $1999-2000$ & 6.408 & 6.102 & 306 & $95,2 \%$ \\
\hline $2000-2001$ & 6.380 & 5.902 & 478 & $92,5 \%$ \\
\hline $2001-2002$ & 6.936 & 6.404 & 532 & $92,3 \%$ \\
\hline $2002-2003$ & 7.467 & 7.036 & 431 & $94,2 \%$ \\
\hline $2003-2004$ & 8.176 & 7.289 & 887 & $89,2 \%$ \\
\hline $2004-2005$ & 6.902 & 5.989 & 913 & $86,8 \%$ \\
\hline $2005-2006$ & 7.159 & 5.969 & 1.190 & $83,4 \%$ \\
\hline $2006-2007$ & 7.150 & 5.355 & 1.795 & $74,9 \%$ \\
\hline $2007-2008$ & 7.302 & 4.872 & 2.430 & $66,7 \%$ \\
\hline Total & 75.795 & 65.854 & 9.941 & $87,3 \%$ \\
\hline
\end{tabular}

Fuente: INE y TESEO. 


\subsection{DIALNET: Tesis Doctorales (http://DIALNET.unirioja.es/servlet/ portadatesis). Universidad de La Rioja (UR)}

Desde el año 2001, DIALNET (Magriñá, 2007) es un sistema de acceso libre a la literatura científica española (artículos, capítulos de obras colectivas, tesis y libros), con la finalidad de darle una máxima difusión (idiomas de consulta: alemán, castellano, catalán, francés, gallego, inglés, portugués y vasco). En DIALNET cooperan 27 bibliotecas universitarias ( 5 de Sudamérica) y 5 bibliotecas públicas o especializadas. También se ofrecen algunos servicios de valor añadido: selección y descarga de resultados; suscripción a un sistema de alertas (con más de 419.000 usuarios registrados); noticias vía RSS (Really Simple Syndication), etc. A partir de 2004, DIALNET cumple con el protocolo OAI-PMH. Respecto a las tesis doctorales, 38 universidades españolas depositan datos bibliográficos en DIALNET, un $76,4 \%$ de los cuales también incorporan el texto completo (Tabla VIII). En la actualidad, se pueden consultar 16.424 tesis por autor, título, universidad, idioma y año, de las cuales 12.541 a texto completo (junio 2009).

\subsection{TDR: Tesis Doctorales en Red (http://www.tdr.cesca.es). Consorci de Biblioteques Universitàries de Catalunya (CBUC) y Centre de Supercomputació de Catalunya (CESCA)}

En funcionamiento desde el año 2001, este depósito cooperativo (Anglada y otros, 2002; Anglada y Reoyo, 2005) facilita el acceso abierto al texto íntegro de 6.064 tesis doctorales aprobadas en 12 universidades catalanas, además de 1.353 correspondientes a 8 instituciones de enseñanza superior del resto de España (Tabla VIII). Desde el primer semestre de 2008, TDR registró un crecimiento cercano a las 2.500 tesis, alcanzando los 7.417 registros actuales. Desde finales del año 2003, TDR es proveedor y recolector de metadatos mediante el protocolo OAI-PMH, hecho que le ha permitido aumentar considerablemente las consultas recibidas. Este depósito se puede consultar en castellano, catalán, gallego e inglés a partir de dos opciones:

- Búsqueda exclusiva en TDR, con múltiples criterios: autor, director, título, universidad, departamento, materia de la Clasificación Decimal Universal (CDU), palabras clave, texto libre y año.

- Búsqueda simultánea y global a 13 depósitos científicos (cooperativos e institucionales) por autor, título, texto libre y año. Esta posibilidad está activada desde mayo de 2007 y permite la consulta a más 18.000 tesis doctorales españolas a texto completo, correspondientes a 39 universidades españolas. 
TABLA VIII

Comparativa por universidades de las tesis registradas por INE, TESEO,

DIALNET y TDR (España 1997-2008)

\begin{tabular}{|c|c|c|c|c|c|c|c|c|}
\hline Universidades & $\begin{array}{l}\text { INE* }^{*} \\
\text { Tesis }\end{array}$ & $\begin{array}{l}\text { TESEO* } \\
\text { Tesis }\end{array}$ & $\begin{array}{c}\% \\
\text { TESEO } \\
\text { resp. } \\
\text { INE }\end{array}$ & $\begin{array}{l}\text { DIAL- } \\
\text { NET } \\
\text { Tesis }\end{array}$ & $\begin{array}{l}\text { DIAL- } \\
\text { NET } \\
\text { Tesis } \\
\text { TC }^{* *}\end{array}$ & $\begin{array}{c}\text { DIAL- } \\
\text { NET } \\
\% \\
\text { Tesis TC }\end{array}$ & $\begin{array}{l}\text { TDR } \\
\text { Tesis }\end{array}$ & $\begin{array}{l}\text { TDR } \\
\text { Global }\end{array}$ \\
\hline $\begin{array}{l}\text { U. Alfonso X El Sa- } \\
\text { bio }\end{array}$ & 10 & 5 & 50,0 & & & & & \\
\hline U. Almería & 523 & 331 & 63,3 & & & & & \\
\hline U. Antonio Nebrija & 10 & 10 & 100,0 & & & & & \\
\hline $\begin{array}{l}\text { U. Autónoma de } \\
\text { Madrid }^{* * * *}\end{array}$ & 4.469 & 3.092 & 69,2 & 235 & 128 & 54,5 & & \\
\hline U. Camilo José Cela & 2 & 1 & 50,0 & & & & & \\
\hline $\begin{array}{l}\text { U. Carlos III de Ma- } \\
\text { drid*** }^{\text {* }}\end{array}$ & 668 & 589 & 88,2 & 135 & 86 & 63,7 & & 156 \\
\hline $\begin{array}{l}\text { U. Católica de Va- } \\
\text { lencia }\end{array}$ & 2 & 2 & 100,0 & & & & & \\
\hline $\begin{array}{l}\text { U. Católica S. Anto- } \\
\text { nio de Murcia }\end{array}$ & 39 & 26 & 66,7 & & & & & \\
\hline $\begin{array}{l}\text { U. Complutense de } \\
\text { Madrid*** }\end{array}$ & 7.544 & 6.726 & 89,2 & 4.542 & 4.170 & 91,8 & & 6.980 \\
\hline U. de Alcalá**** & 1.388 & 1.214 & 87,5 & 102 & 65 & 63,7 & & 111 \\
\hline U. de Burgos**** & 235 & 220 & 93,6 & 27 & 23 & 85,2 & & 23 \\
\hline U. de Cádiz & 888 & 588 & 66,2 & & & & & \\
\hline U. de Cantabria & 618 & 547 & 88,5 & 171 & 67 & 39,2 & 66 & \\
\hline $\begin{array}{l}\text { U. de Cardenal He- } \\
\text { rrera-CEU }\end{array}$ & 57 & 45 & 78,9 & & & & & \\
\hline $\begin{array}{l}\text { U. de Castilla-La } \\
\text { Mancha }\end{array}$ & 626 & 652 & 104,2 & & & & & \\
\hline U. de Córdoba ${ }^{* * * *}$ & 1.405 & 1.190 & 84,7 & 35 & 4 & 11,4 & & \\
\hline U. de Deusto & 414 & 371 & 89,6 & & & & & \\
\hline U. de Extremadura & 879 & 955 & 108,6 & 230 & 157 & 68,3 & & \\
\hline U. de Granada**** & 3.452 & 2.845 & 82,4 & & & & & \\
\hline U. de Huelva**:* & 272 & 227 & 83,5 & 29 & 27 & 93,1 & & 33 \\
\hline U. de Jaén & 389 & 297 & 76,3 & & & & & \\
\hline U. de La Laguna & 1.038 & 958 & 92,3 & 674 & 489 & 72,6 & & \\
\hline U. de La Rioja & 173 & 163 & 94,2 & 197 & 40 & 20,3 & & \\
\hline $\begin{array}{l}\text { U. de Las Palmas de } \\
\text { Gran Canaria**** }\end{array}$ & 836 & 718 & 85,9 & 184 & 115 & 62,5 & & \\
\hline
\end{tabular}


TABLA VIII (continuación)

\begin{tabular}{|c|c|c|c|c|c|c|c|c|}
\hline Universidades & $\begin{array}{l}\text { INE* }^{*} \\
\text { Tesis }\end{array}$ & $\begin{array}{l}\text { TESEO* } \\
\text { Tesis }\end{array}$ & $\begin{array}{c}\% \\
\text { TESEO } \\
\text { resp. } \\
\text { INE }\end{array}$ & $\begin{array}{l}\text { DIAL- } \\
\text { NET } \\
\text { Tesis }\end{array}$ & $\begin{array}{l}\text { DIAL- } \\
\text { NET } \\
\text { Tesis } \\
\text { TC** }\end{array}$ & $\begin{array}{c}\text { DIAL- } \\
\text { NET } \\
\% \\
\text { Tesis TC }\end{array}$ & $\begin{array}{l}\text { TDR } \\
\text { Tesis }\end{array}$ & $\begin{array}{l}\text { TDR } \\
\text { Global }\end{array}$ \\
\hline U. de León & 752 & 695 & 92,4 & 15 & 1 & 6,7 & & \\
\hline U. de Málaga & 1.463 & 1.390 & 95,0 & 320 & 177 & 55,3 & & \\
\hline $\begin{array}{l}\text { U. de Miguel Her- } \\
\text { nández de Elche }\end{array}$ & 629 & 494 & 78,5 & & & & & \\
\hline U. de Mondragón & 48 & 41 & 85,4 & & & & & \\
\hline U. de Murcia**** & 1.729 & 1.579 & 88,1 & 227 & 122 & 53,7 & 206 & \\
\hline U. de Navarra**** & 1.806 & 1.275 & 70,6 & & & & & \\
\hline U. de Oviedo & 1.786 & 1.593 & 89,2 & 185 & 61 & 33,0 & 59 & \\
\hline U. de Salamanca ${ }^{* * *}$ & 2.397 & 1.502 & 62,7 & 166 & 83 & 50,0 & & \\
\hline U. de Sevilla & 3.403 & 2.109 & 62,0 & & & & & \\
\hline U. de Valladolid*** & 1.452 & 1.103 & 76,0 & & & & & \\
\hline U. de Zaragoza**** & 2.060 & 1.520 & 73,8 & 254 & 15 & 5,9 & 58 & 88 \\
\hline U. del País Vasco & 2.308 & 2.375 & 102,9 & & & & & \\
\hline $\begin{array}{l}\text { U. Europea de Ma- } \\
\text { drid }\end{array}$ & 65 & 19 & 29,2 & & & & & \\
\hline $\begin{array}{l}\text { U. Nacional de Edu- } \\
\text { cación a Distan- } \\
\text { cia*** }^{* * *}\end{array}$ & 1.346 & 1.424 & 105,8 & & & & & \\
\hline U. Pablo de Olavide & 203 & 191 & 94,1 & & & & & \\
\hline $\begin{array}{l}\text { U. Politécnica de } \\
\text { Cartagena**** }\end{array}$ & 188 & 178 & 94,7 & 8 & 3 & 37,5 & & 60 \\
\hline $\begin{array}{l}\text { U. Politécnica de } \\
\text { Madrid**** }\end{array}$ & 1.858 & 1.936 & 104,2 & 1.148 & 632 & 55,1 & & 852 \\
\hline $\begin{array}{l}\text { U. Pontificia de Co- } \\
\text { millas }\end{array}$ & 315 & 307 & 97,5 & & & & & \\
\hline $\begin{array}{l}\text { U. Pontificia de Sa- } \\
\text { lamanca }\end{array}$ & 326 & 150 & 46,0 & & & & & \\
\hline $\begin{array}{l}\text { U. Pública de Nava- } \\
\text { rra }\end{array}$ & 412 & 344 & 83,5 & 40 & 3 & 7,5 & & \\
\hline $\begin{array}{l}\text { U. Rey Juan Car- } \\
\operatorname{los}^{* * * *}\end{array}$ & 321 & 218 & 67,9 & 56 & 54 & 96,4 & & 65 \\
\hline U. San Pablo-CEU & 228 & 176 & 77,2 & & & & & \\
\hline U. SEK & 6 & 0 & 0,0 & & & & & \\
\hline U. da Coruña**** & 762 & 700 & 91,9 & 67 & 20 & 29,9 & 23 & \\
\hline
\end{tabular}


TABLA VIII (continuación)

\begin{tabular}{|c|c|c|c|c|c|c|c|c|}
\hline Universidades & $\begin{array}{l}\text { INE* } \\
\text { Tesis }\end{array}$ & $\begin{array}{c}\text { TESEO* } \\
\text { Tesis }\end{array}$ & $\begin{array}{c}\% \\
\text { TESEO } \\
\text { resp. } \\
\text { INE }\end{array}$ & $\begin{array}{l}\text { DIAL- } \\
\text { NET } \\
\text { Tesis }\end{array}$ & $\begin{array}{c}\text { DIAL- } \\
\text { NET } \\
\text { Tesis } \\
\text { TC** }\end{array}$ & $\begin{array}{c}\text { DIAL- } \\
\text { NET } \\
\% \\
\text { Tesis TC }\end{array}$ & $\begin{array}{c}\text { TDR } \\
\text { Tesis }\end{array}$ & $\begin{array}{l}\text { TDR } \\
\text { Global }\end{array}$ \\
\hline $\begin{array}{l}\text { U. de Santiago de } \\
\text { Compostela }\end{array}$ & 2.299 & 1.918 & 83,4 & & & & & \\
\hline U. de Vigo & 849 & 797 & 93,9 & & & & & \\
\hline U. Abat Oliba-CEU & 21 & 0 & 0,0 & & & & 9 & \\
\hline $\begin{array}{l}\text { U. Autònoma de } \\
\text { Barcelona }^{* * *}\end{array}$ & 3.484 & 3.102 & 89,0 & 1.929 & 1.747 & 90,6 & 2.219 & \\
\hline U. d'Alacant**** & 900 & 822 & 91,3 & 555 & 541 & 97,5 & & 824 \\
\hline U. de Barcelona**** & 5.312 & 3.507 & 66,0 & 1.340 & 1.105 & 82,5 & 1.529 & \\
\hline U. de Girona*** & 431 & 418 & 97,0 & 270 & 266 & 98,5 & 355 & \\
\hline $\begin{array}{l}\text { U. de les Illes Ba- } \\
\text { lears }\end{array}$ & 421 & 230 & 54,6 & 77 & 45 & 58,4 & 69 & \\
\hline U. de Lleida & 434 & 376 & 86,6 & 140 & 137 & 97,9 & 190 & \\
\hline U. de València & 3.631 & 3.009 & 82,9 & 732 & 457 & 62,4 & 698 & \\
\hline U. de Vic & 4 & 2 & 50,0 & 1 & 1 & 100,0 & 2 & \\
\hline $\begin{array}{l}\text { U. Internacional de } \\
\text { Catalunya }\end{array}$ & 68 & 35 & 51,5 & & & & 12 & \\
\hline U. Jaume I*** & 441 & 384 & 87,1 & 125 & 95 & 76,0 & 174 & \\
\hline $\begin{array}{l}\text { U. Oberta de Cata- } \\
\text { lunya }\end{array}$ & 13 & 12 & 92,3 & 2 & 2 & 100,0 & 9 & \\
\hline $\begin{array}{l}\text { U. Politècnica de } \\
\text { Catalunya*** }\end{array}$ & 2.036 & 1.653 & 81,2 & 1.059 & 700 & 66,1 & 872 & \\
\hline $\begin{array}{l}\text { U. Politècnica de } \\
\text { València }^{* * * *}\end{array}$ & 2.142 & 1.812 & 84,6 & 567 & 348 & 61,4 & & 319 \\
\hline U. Pompeu Fabra*** & 459 & 372 & 81,0 & 171 & 171 & 100,0 & 293 & \\
\hline U. Ramon Llull & 336 & 293 & 87,2 & 76 & 59 & 77,6 & 109 & \\
\hline U. Rovira i Virgili & 651 & 522 & 80,2 & 333 & 325 & 97,6 & 465 & \\
\hline TOTAL & 75.795 & 62.355 & 82,3 & 16.424 & 12.541 & 76,4 & 7.417 & 9.511 \\
\hline
\end{tabular}

* Del Curso 1997-1998 al curso 2007-2008.

** Tesis TC $=$ Tesis Texto completo.

*** Universidades que disponen de depósito institucional de acceso abierto.

Fuente: INE, TESEO, DIALNET, TDR y Grupo de investigación "Acceso abierto a la Ciencia". 


\subsection{Panorama internacional}

Al margen de las bases de datos comerciales (DAI Dissertation Abstracts Internacional, UMI Dissertation Publishing, etc.), cabe destacar el proyecto DARTEurope E-theses Portal (http://www.dart-europe.eu) (Moyle, 2008), una asociación de bibliotecas de investigación y de consorcios bibliotecarios que trabajan conjuntamente para la mejora del acceso global y centralizado de las tesis doctorales europeas. En la actualidad, DART-Europe agrupa 98.398 tesis doctorales procedentes de 11 países: Alemania, Bélgica, España, Estonia, Finlandia, Hungría, Irlanda, Noruega, Reino Unido, Suecia y Suiza. Se trata de un servicio con algunas funcionalidades todavía en desarrollo y, a nivel español, básicamente incluye las universidades representadas en el CBUC. La búsqueda avanzada presenta las siguientes opciones: autor, título, resumen, año, colección (repositorio, grupo de repositorios o proveedor de servicio), país, institución e idioma de la tesis.

A su vez, DART-Europe es el grupo de trabajo europeo de la red internacional Networked Digital Library of Theses and Dissertations (NDLTD) (http://www. ndltd.org). En funcionamiento desde el año 1997, NDLTD constituye un referente de proyecto distribuido que ofrece servicios comunes a todos los socios federados, los cuales operan de manera autónoma. Como reconocimiento a su labor, NDLTD colabora con la UNESCO en la redacción y actualización de la Guía de tesis y disertaciones electrónicas (http://www.etdguide.bibliored.cl). Este documento tiene la finalidad de impulsar las bibliotecas digitales de tesis doctorales en el marco de la cooperación internacional (Orera, 2003a).

Otros servidores internacionales con presencia de tesis españolas son $C y$ bertesis: tesis electrónicas en linea (Universidad de Santiago de Chile) (http:// www.cybertesis.net) y Cyberthèses: portail francophone des thèses électroniques (Université Lumière de Lyon 2 y Université de Montréal) (http://cyberdocs.univlyon2.fr).

\subsection{Análisis de los datos de las bases de datos y repositorios cooperativos nacionales e internacionales}

Como se puede observar, la base de datos TESEO adolece de múltiples carencias, y aunque puede ser un instrumento de control y difusión muy útil, se tienen que solucionar algunas de sus deficiencias. Entre ellas:

- Exhaustividad: a partir de la comparación con los datos del INE desde el curso 1997-1998 hasta el 2007-2008 (Tabla VII), se observa como TESEO recopila el 87,3\% de las tesis doctorales aprobadas en España.

- Calidad: la información resulta bastante desigual (Agudelo y otros, 2003; Delgado y otros, 2006) y se detectan campos sin rellenar, errores manifiestos, palabras clave imprecisas, falta de actualización, etc. Por este motivo y 
de cara a los análisis posteriores, el diseño de las consultas y los datos extraídos deben ser revisados por expertos.

- Texto completo: se está trabajando para solucionar este déficit.

- Interacción: habría que facilitar la conexión ágil entre los usuarios y los propios investigadores a partir de datos de contacto actualizados.

- Idioma: la pantalla de consulta sólo está en castellano.

Un elemento fundamental seria conseguir que todas las universidades envíen información regularmente para que se pueda introducir en la base de datos TESEO.

Respecto a los depósitos cooperativos, la Estadística de enseñanza universitaria registra 67 universidades con alumnos matriculados en doctorado (48 públicas y 19 privadas), de los cuales 40 (35 públicas y 5 privadas) están presentes a través de DIALNET y TDR (búsqueda específica o global). A su vez, desde la entrada en funcionamiento en 2001 de estos repositorios, el INE ha contabilizado 51.092 tesis aprobadas (del curso 2001-2002 al 2007-2008), más de un tercio de las cuales, aproximadamente, son accesibles mediante estos dos recursos. El número de documentos depositados está creciendo de modo acelerado y cabe la posibilidad de plantearse proyectos de digitalización de las colecciones retrospectivas.

Además, algunas universidades españolas están ya desarrollando sistemas para difundir y acceder libremente a distintos documentos entre ellos, las tesis doctorales, y potenciando la realización de proyectos de sistemas abiertos (Open Acces) con múltiples aplicaciones.

También a nivel europeo se observa esta tendencia y un ejemplo importante como se ha apuntado anteriormente es el grupo de trabajo DART-Europe.

Así pues, a partir del análisis de los datos estadísticos y de los recursos descritos, cabe plantearse el grado de accesibilidad de las tesis doctorales en España, a partir de la exhaustividad y actualización de las fuentes, la presencia del texto completo, los sistemas de acceso abierto, la colaboración entre distintos organismos, universidades, grupos de trabajo, proyectos conjuntos, etc.

Al analizar los datos de la comparativa por universidades de las tesis registradas por INE, TESEO, DIALNET y TDR (Tabla VIII) se pueden resaltar algunos aspectos significativos.

INE y TESEO recogen los datos de las universidades tanto públicas como privadas, en cambio DIALNET y TDR solo algunas públicas. El INE contabiliza, en general, más tesis doctorales que TESEO (Tabla VII), debido a factores ya apuntados anteriormente, criterios de recogida de datos, períodos de tiempo: años naturales, cursos académicos, etc., a excepción de las Universidades de Castilla-La Mancha, Extremadura, País Vasco, a Distancia y Pontificia de Comillas. (Como puede observarse el porcentaje del número de tesis de TESEO respecto al INE, sobrepasa el $100 \%$ en las universidades citadas). A su vez, mientras el número total de tesis registradas por el INE (75.795) coincide totalmente con el de la Tabla VII, el total de tesis registradas en TESEO presenta una pequeña variación de un 5,3\% (65.854 en la Tabla VII y 62.355 en la Tabla VIII), quizá 
debido a que no se han contabilizado algunas de las tesis registradas en algunas de las universidades (Universidad SEK o Universidad Abat Oliba-CEU con 0 tesis, por ejemplo).

En los datos facilitados en DIALNET, se observa que el $100 \%$ de las tesis registradas son a texto completo en las universidades de Vic, Politècnica de Catalunya y Pompeu Fabra, superior al 90\% en las universidades Complutense de Madrid, Huelva, Rey Juan Carlos, Autónoma de Barcelona, Alacant, Girona y de Lleida. (Ver ${ }^{(* *)}$ Tabla VIII.)

Finalmente cabe destacar que de las 67 universidades comparadas, 27 de ellas públicas, disponen de un depósito institucional de acceso abierto, (Ver (***) Tabla VIII.)

\section{Conclusiones y consideraciones finales}

Algunas conclusiones ya se han ido aportando en los apartados de análisis de los datos tanto de las fuentes estadísticas (4), como de las bases de datos y repositorios cooperativos (5). Por ello en este apartado se comentaran tan solo las más generales y algunas consideraciones con proyección de futuro.

Los objetivos propuestos para este artículo: a) recopilar y analizar los datos estadísticos fundamentales sobre tesis doctorales aprobadas en España en el período 1997-2008, y b) describir algunas de las principales bases de datos y recursos cooperativos nacionales e internacionales usados para difundir específicamente las investigaciones doctorales españolas, se han cumplido, aunque con algunos pequeños desajustes según la procedencia de los datos. La comparación de los datos estadísticos con las fuentes especializadas ha permitido observar la determinación del sistema académico español para formar nuevos investigadores, así como el grado de accesibilidad a las tesis doctorales.

En términos cuantitativos, la formación de nuevos investigadores en España resulta claramente inferior a la media europea. Un sistema científico consolidado no puede ofrecer unos indicadores tan negativos en la formación de científicos. En futuras investigaciones cabría estudiar detalladamente las posibles causas (tasa elevada de abandonos, duración dilatada en el tiempo, etc.) para intentar revertir esta situación y plantear soluciones, como apuntan diversos autores:

- Mejora de la consideración científica, social y económica del doctorado, así como del título de doctor y de la tarea de profesores y directores (Agudelo y otros, 2003; López y otros, 2005b).

- Aumento del número de becarios predoctorales y del apoyo a proyectos de investigación (Agudelo y otros, 2003).

- Diseño de una carrera investigadora con atractivas perspectivas de futuro (López y otros, 2005a).

- Fomento de la cultura de la calidad y de la movilidad. En este sentido, es necesario conocer el impacto científico a posteriori de las tesis doctorales 
(por ejemplo, mediante artículos en revistas internacionales). La existencia de información sólida y transparente permite determinar la eficacia de estas investigaciones como factor de promoción de la ciencia y detectar focos, redes y escuelas (Moyano y otros, 2006; López y otros, 2005a; Figueredo y otros, 2002).

Las actuaciones científicas y tecnológicas generan nuevos conocimientos, los cuales se comunican mediante documentos. De esta forma, la comunidad científica revisa los contenidos y crea las bases para futuros descubrimientos. Actualmente, una prioridad básica de la comunicación científica consiste en la transmisión rápida y ágil del conocimiento entre los grupos de investigadores. En este sentido, la combinación de TIC, acceso abierto y fuentes documentales especializadas facilita la visibilidad internacional de las tesis doctorales, documentos de difícil acceso hace tan sólo pocos años. En última instancia, se trata de garantizar la diseminación global del nuevo conocimiento, condición esencial para la formación de nuevos investigadores y para el desarrollo general de la ciencia.

En el campo de la comunicación y documentación científicas, podemos apuntar algunas de las tendencias con mayor proyección de futuro:

- Cooperación e interconexión de los sistemas mediante protocolos estándar (por ejemplo, OAI-PMH) para el establecimiento de redes de conocimiento accesibles mediante pantallas de consulta global, con diversidad de idiomas y criterios de búsqueda.

- Acceso a volúmenes masivos de información, hasta consultar el texto completo de los documentos de interés para el usuario.

- Potenciación de los servicios de valor añadido, con el objetivo final de mejorar el flujo de conocimiento entre emisores y receptores.

- Peso cada vez mayor del acceso abierto a contenidos científicos, a partir del respeto a las condiciones establecidas en cada caso por los agentes implicados (autores, editores, proveedores, bibliotecas, etc.).

- Establecimiento de un sistema que garantice la preservación y conservación de la información científica a largo plazo.

\section{Bibliografía}

Agudelo, D.; Bretón-López, J.; Ortiz-Recio, G.; Poveda-Vera, J.; Teva, I.; Valor-Segura, I., y Vico, C. (2003): Análisis de la productividad científica de la psicología española a través de las tesis doctorales. Psicothema, vol. 15 (4), 595-609.

Alcaín, M. D., y Ruiz-Galvez, P. M. (1997): Los estudios bibliométricos de la base de datos Dissertation Abstracts On Disc (1981-1995): Revista General de Información y Documentación, vol. 7 (2), 167-179.

Anglada, L.; Bárcena, I.; Cambras, J.; Comellas, N., y Huguet, M. (2002): Acceso electrónico a las tesis doctorales de Cataluña. El Profesional de la Información, vol. 11 (1), 28-33. 
Anglada, L.; Reoyo, S. (2005): Els dipòsits electrònics col-lectius del CBUC: antecedents i situació actual. Item: revista de biblioteconomia i documentació, no ${ }^{\circ} 41,55-65$.

Barbosa, A. (2000): Diez años de investigación ornitológica en España a través de las tesis doctorales. Revista Ibérica de Ornitología, vol. 47 (2), 273-278.

Bonal Zazo, J. L. (2000): La investigación universitaria sobre archivos y archivística en España a través de las tesis doctorales. En: Primer Congreso Universitario de Ciencias de la Documentación: Teoría, historia y metodología de las ciencias de la documentación, 351-358. Madrid: Universidad Complutense de Madrid.

Buela-Casal, G. (2005): An Overview of Scientific Productivity of Spanish Universities". International Journal of Clinical and Health Psychology, vol. 5 (1), 175-190.

Castillo, A., y Xifra, J. (2006): Investigación bibliométrica de las tesis doctorales españolas sobre relaciones públicas. Anàlisi: quaderns de comunicació i cultura, nº 34, 141-161.

Centeno, C.; Hernansanz, S.; Flores, L. A.; López-Lara, F.; Rubiales, A., y Flores, L. A. (2001): Tesis doctorales relacionadas con cuidados paliativos realizadas en la universidad española entre 1985 y 2000. Medicina Paliativa, vol. 8 (4), 181-189.

Civera Molla, C., y Tortosa Gil, F. (2001): Estado de la investigación psicológica en España: el grado de doctor y la investigación académica (1976-1998): Papeles del Psicólogo, $\mathrm{n}^{\circ} 79,42-52$.

Delgado López-Cózar, E.; Torres-Salinas, D.; Jiménez-Contreras, E., y Ruiz-Pérez, R. (2006): Análisis bibliométrico y de redes sociales aplicado a las tesis bibliométricas defendidas en España (1976-2002): temas, escuelas científicas y redes académicas. Revista Española de Documentación Científica, vol. 29 (4), 493-524.

Diego, J. I., y Prim, M. P. (2008): Análisis de las tesis doctorales en otorrinolaringología presentadas en España en el período 1976-2005. Acta Otorrinolaringológica Española, vol. 59 (6), 292-297.

Fernández Cano, A.; Torralba, M., y Vallejo, M. (2008): Previsión y prospectiva de la producción española de tesis doctorales de pedagogía (1976-2002). Revista de Investigación Educativa, vol. 26 (1), 191-208.

Fernández Cano, A.; Torralbo, M.; Rico, L.; Gutiérrez, P., y Maz, A. (2003): Análisis cienciométrico de las tesis doctorales españolas en educación matemática. Revista Española de Documentación Científica, vol. 26 (2), 162-176.

Fernández, M. (2004): Los estudios españoles sobre adopción y acogimiento familiar 19742004. Boletín de Psicología, no 81, 7-31.

Figueredo, E.; Sánchez Perales. G.; Villalonga, A., y Castillo, J. (2002): Tesis doctorales españolas sobre anestesiología y publicaciones científicas de sus autores. Revista Española de Anestesiología y Reanimación, vol. 49 (3), 124-130.

Fuentes Pujol, M. E., y González Quesada, A. (2002): Tesis doctorales en España en información y documentación. En Morán Suárez, M. A., y Rodríguez López, M. (eds.), La Documentación para la investigación: homenaje a José Antonio Martín Fuertes, León, Universidad de León, 229-241.

Gete Benavente, P. (2005): La historia de la farmacia en la España del siglo XIX a través de las tesis doctorales que tratan del tema y que se conservan en el Archivo de la Universidad Complutense de Madrid. Tesis doctoral, Universidad de Alcalá de Henares.

Guisan, C., y Expósito, P. (2009): Fifty years of Econometrics Research in Spain: Doctoral Dissertations, international publications and other contributions, 1956-2006. Disponible en http://ideas.repec.org/p/eaa/ecodev/98.html [consulta 15 de enero de 2009]. 
Hernández Borge, J. (1999): Cuarenta años de tesis doctorales en geografía en la Universidad de Santiago de Compostela (1959-1998). Estudios Geográficos, vol. 60 (237), 727-740.

Jones D. E., y Baró J. (1997): Tesis doctorals i treballs de recerca universitaris sobre comunicació als Països Catalans 1954-1996: aproximació bibliomètrica. Anàlisi: quaderns de comunicació $i$ cultura, 157-187.

Lázaro Torres, M. L. (2002): La geografía a las puertas del tercer milenio a partir de las tesis doctorales leídas en los noventa. Estudios Geográficos, vol. 63 (246), 154-168.

López Yepes, J. (2002a): Focos de investigación y escuelas científicas en documentación: la experiencia de las tesis doctorales. El Profesional de la Información, vol. 11 (1), 46-51.

López Yepes, J. (2002b): Focos de investigación y escuelas científicas en documentación a través de la realización y dirección de tesis doctorales: el caso del Departamento de Biblioteconomía y Documentación de la Universidad Complutense de Madrid (19832001). Documentación de las Ciencias de la Información, $\mathrm{n}^{\circ}$ 25, 19-54.

López Yepes, J.; Fernández Bajón, M. T., y Prat Sedeño, J. (2005a): La investigación española en documentación informativa a examen en Ibersid 2004. Estado de la cuestión con especial referencia a las tesis doctorales (1976-2004). El Profesional de la Información, vol. 14 (1), 50-57.

López Yepes, J.; Fernández Bajón, M. T., y Prat Sedeño, J. (2005b): Las tesis doctorales en biblioteconomía y documentación: diagnóstico y propuesta de criterios de evaluación. Documentación de las Ciencias de la Información, $\mathrm{n}^{\circ}$ 28, 173-187.

Magriñá Contreras, M. (2007): La difusión de la producción científica española a través del proyecto DIALNET. Boletín de la ANABAD, vol. 57 (4), 9-22.

Martínez Cerverón, R. (2007): Maestros y discípulos en la psicología contemporánea: un estudio a través de las tesis doctorales. Tesis doctoral, Universidad de Valencia, 2007.

Martínez Pestaña, M. J. (2004): La producción de tesis doctorales sobre temas publicitarios (1971-2001). Documentación de las Ciencias de la Información, n 27, 237-267.

Melero, R.; Lopez Medina, A., y Prats, J. (2008): Landscape of Open Access Institutional Repositories in Spain. Third International Conference on Open Repositories 2008, 1-4 April 2008, Southampton, United Kingdom. Disponible en: http://pubs.or08.ecs.soton. ac.uk/56/ [consulta 15 de enero de 2009].

Merlo Vega, J. A., y Sorli Rojo, A. (2002): Bases de datos y recursos en Internet sobre tesis doctorales. Revista Española de Documentación Científica, vol. 25 (1), 95-106.

Miguel Alonso, A. (2000): Aportaciones al estudio de la literatura gris universitaria: La evolución de la tesis doctoral en España. Primer Congreso Universitario de Ciencias de la Documentación: Teoría, historia y metodología de las ciencias de la documentación, 645-651. Madrid: Universidad Complutense de Madrid.

Moralejo Álvarez, M. R. (2000): Las tesis doctorales de las universidades españolas: control bibliográfico y acceso. Revista General de Información y Documentación, vol. 10 (1), 235-243.

Moyano, M.; Delgado Domínguez, C., y Buela Casal, G. (2006): Análisis de la productividad científica de la Psiquiatría española a través de las tesis doctorales en la base de datos TESEO (1993-2002). International Journal of Psychology and Psychological Therapy, 2006, vol. 6 (1), 111-120. 
Moyle, M. (2008): Improving Access to European E-theses: the DART-Europe Programme. LIBER Quarterly, 2008, vol. 18 (3/4), 413-423.

Muñoz Muñoz, A. (2005): The Scholarly Transition of Female Academics at the University of Granada (1975-1990). Scientometrics, vol. 64 (3), 325-350.

Muñoz Tinoco, C., y Peña Arrebola, A. (1998): Tesis doctorales sobre rehabilitación presentadas en España en el período 1976-1996. Rehabilitación, vol. 32 (4), 221-224.

Muñoz Tinoco, C., y Peña Arrebola, A. (2000): Tesis doctorales sobre reumatología presentadas en España entre 1976 y 1997. Estudio bibliométrico. Revista Española de Reumatología, vol. 3 (27), 93-98.

Nagore Ferrer, M. (2005): Tesis doctorales. La investigación musical en España: situación actual y perspectivas de futuro. Revista de Musicología, vol. 28 (2), 1451-1470.

Orera Orera, L. (2003a): Bibliotecas digitales de tesis doctorales: Metodología para su planificación. Boletín de la Asociación Andaluza de Bibliotecarios, 2003, n 72, 55-72.

Orera Orera, L. (2003b): La edición digital de tesis doctorales: hacia la resolución de los problemas de accesibilidad. Revista Interamericana de Bibliotecología, vol. 26 (1), 11-35.

Orera Orera, L. (2004): Las tesis sobre biblioteconomía y bibliotecas y su accesibilidad a través de las bibliotecas universitarias españolas. En Fernández Bajón, M. T.; López López, P., y López Yepes, J. (eds.), Estudios de biblioteconomía y documentación. Homenaje a la profesora María Rosa Garrido Arilla. Madrid, Universidad Complutense de Madrid, 91-102.

Ortega Martínez, E., y Rodríguez Herráez, B. (2004): La investigación turística a través de tesis doctorales. Un análisis comparativo entre España y Francia. Estudios turísticos, $\mathrm{n}^{\circ} 159,5-27$.

Pablo Núñez, L. (2006): Aproximación bibliográfica a los estudios de semántica estructural en España. Hesperia: anuario de filología hispánica, vol. 9, 163-181.

Pérez López, I. (2007): Historia antigua y ciencias afines en las publicaciones periódicas $y$ tesis doctorales del último tercio del siglo XIX español (1868-1900). Tesis doctoral, Universidad de Málaga.

Ponce de León Elizondo, A.; Gargallo Ibort, E., y Loza Olave, E. (1998): Análisis de las tendencias en las tesis doctorales de educación física. Cursos 1980-1981 a 1995-1996. Apunts, $\mathrm{n}^{\circ}$ 52, 104-108.

Rorralbo Rodríguez, M. (2001): Análisis cientimétrico, conceptual y metodológico de las tesis doctorales españolas en educación matemática 1976-1998. Tesis doctoral, Universidad de Granada.

Sabater Lorenzo, P. (2001): Las tesis doctorales de las facultades de ciencias de la Universidad de Murcia, 1955-1990: catálogo, estadística descriptiva y bibliometría. Tesis doctoral, Universidad de Murcia.

Torres Ramírez, I., y Torres Salinas, D. (2005): Tesis doctorales sobre estudios de las mujeres en España (1976-2002). A propósito de un indicador definitivo en investigación. Revista Española de Documentación Científica, vol. 28 (4), 479-499.

Urbano, C. (2000a): El análisis de citas en publicaciones de usuarios de bibliotecas universitarias: estudio de las tesis doctorales en informática de la Universidad Politécnica de Cataluña, 1996-1998. Tesis doctoral, Universidad de Barcelona, 2000.

Urbano, C. (2000b): Tipologia documental citada en tesis doctorals d'informàtica: bases empíriques per a la gestió equilibrada de colleccions. BiD: textos universitaris de bi- 
blioteconomia $i$ documentació, $\mathrm{n}^{\circ}$ 5. Disponible en: http://www.ub.edu/biblio/ bid/05urban1.htm [consulta 15 de enero de 2009].

Vallejo Ruiz, M.; Fernández Cano, A., y Torralbo Rodríguez, M. (2006): Patrones de citación en la investigación española en educación matemática. Revista española de documentación científica, vol. 29 (3), 382-397.

Vallmitjana, N., y Sabaté, L. G. (2008): Citation Analysis of Ph.D. Dissertation References as a Tool for Collection Management in an Academic Chemistry Library. College $\varepsilon$ Research Libraries, vol. 69 (1), 72-81.

Villarroya, A. (2008): PhD Theses in Spain: a Gender Study covering the Years 1990-2004. Scientometrics, vol. 77 (3), 469-483.

Zapico Alonso, F. (2002): La investigación en documentación: análisis bibliométrico de las tesis en Documentación. Tesis doctorales en España en información y documentación. En Morán Suárez, M. A., y Rodríguez López, M. (eds.), La Documentación para la investigación: homenaje a José Antonio Martín Fuertes. 2002, León, Universidad de León, 689-713.

\section{Anexo: siglas y acrónimos}

\begin{tabular}{|c|c|}
\hline CBUC & Consorci de Biblioteques de Catalunya. \\
\hline CD-ROM & Compact Disc-Read Only Memory. \\
\hline CDU & Clasificación Decimal Universal. \\
\hline CESCA & Centre de Supercomputació de Catalunya. \\
\hline CRUE & Conferencia de Rectores de Universidades Españolas. \\
\hline CSIC & Consejo Superior de Investigaciones Científicas. \\
\hline CUC & Consejo de Coordinación Universitaria. \\
\hline CYBERTESIS & $\begin{array}{l}\text { Tesis electrónicas en línea (Universidad de Santiago de } \\
\text { Chile). }\end{array}$ \\
\hline CYBERTHESES & $\begin{array}{l}\text { Portail francophone des thèses electroniques (Université } \\
\text { Lumière de Lyon } 2 \text { y Université de Montréal). }\end{array}$ \\
\hline DAI & Dissertation Abstracts Internacional. \\
\hline DART-EUROPE & Digital Access to Research Theses. \\
\hline DIALNET & Portal de diffusion de la producción hispana. \\
\hline EEES & Espacio Europeo de Educación Superior. \\
\hline EHEA & European Higher Education Area. \\
\hline E-LIS & E-Prints in Library and Information Science. \\
\hline EUROSTAT & Statistical Office of the European Communities. \\
\hline ICT & Information and Communication Technologies. \\
\hline INE & Instituto Nacional de Estadística. \\
\hline ISCED & International Standard Classification of Education. \\
\hline
\end{tabular}


ISI

LISTA

MICINN

NDLTD

OAI-PMH

OAIster

OCDE

OPEN ACCESS

OpenDOAR

RD

RSS

TDR

TESEO

TIC

UAB

UB

UE27

UNESCO

UR

UV
Institute for Scientific Information (BDS recogidas en web of knowledge).

Library, Information Science \& Technology Abstracts.

Ministerio de Ciencia e Innovación.

Networked Digital Library of Theses and Dissertations.

Open Archives Initiative Protocol for Metadata Harvesting. Union Catalog of Digital Resources. Buscador académico Internacional.

Organización para la Cooperación y el Desarrollo Económico.

Acceso abierto.

Directory of Open Access Repositories.

Real decreto.

Really Simple Syndication.

Tesis Doctorales en Red.

Base de datos de tesis doctorales españolas.

Tecnologías de la Información y de las Comunicaciones.

Universitat Autònoma de Barcelona.

Universitat de Barcelona.

Union Europea.

Organización de las Naciones Unidas para la Educación, la Ciencia y el Desarrollo.

Universidad de La Rioja.

Universitat de València. 